\title{
Space-charge effects in liquid argon ionization chambers
}

\author{
J.Rutherfoord*, R.B. Walker* \\ Department of Physics, University of Arizona, Tucson, AZ 85721 USA
}

\begin{abstract}
We have uniformly irradiated liquid argon ionization chambers with betas from high-activity Strontium-90 sources. The radiation environment is similar to that in the liquid argon calorimeters which are part of the ATLAS detector installed at CERN's Large Hadron Collider (LHC). We measured the resulting ionization current over a wide range of applied potential for two different source activities and for three different chamber gaps. These studies provide operating experience at exceptionally high ionization rates. In particular they indicate a stability at the $0.1 \%$ level for these calorimeters over years of operation at the full LHC luminosity when operated in the normal mode at an electric field $\mathcal{E}=1.0 \mathrm{kV} / \mathrm{mm}$. We can operate these chambers in the normal mode or in the space-charge limited regime and thereby determine the transition point between the two. This transition point is parameterized by a positive argon ion mobility of $\mu_{+}=0.08 \pm 0.02 \mathrm{~mm}^{2} / \mathrm{Vs}$ at a temperature of $88.0 \pm 0.5 \mathrm{~K}$ and at a pressure of $1.02 \pm 0.02$ bar. In the space-charge limited regime the ionization currents are degraded and show signs of instability. At the highest electric fields in our study $(6.7 \mathrm{kV} / \mathrm{mm})$ the ionization current is still slowly rising with increasing electric field.
\end{abstract}

Keywords: Liquid Argon Calorimetry, Space-charge effects, Noble Liquid Ionization Chambers

PACS: $29.40 . \mathrm{Vj}$

\footnotetext{
${ }^{*}$ Corresponding author

Email address: rutherfo@physics.arizona.edu (J.Rutherfoord)
}

Preprint submitted to Nuclear Instruments and Methods A

December 17, 2014 


\section{Introduction}

3

At the Large Hadron Collider (LHC) [1] at CERN, the two general purpose detectors, ATLAS [2] and CMS [3], are searching for new, fundamental interactions in proton-proton collisions at very high energies. These new processes are expected to be extremely rare. On the other hand, more ordinary proton-proton collisions have large cross sections which rise slowly with energy. The detectors must therefore be capable of picking out the very rare but interesting processes in the midst of a large number of ordinary processes. At the LHC energy and luminosity, these ordinary and plentiful proton-proton collisions produce a large number of relatively low energy particles which hit the detector at high rates.

The ATLAS detector includes liquid argon sampling calorimeters to measure the energies of the particles produced in the proton-proton collisions. A major component of these calorimeters is a dense metal in which each incident particle produces a shower of particles. That is, the relativistic collision products interact with the nuclei of the dense metal of the calorimeter and produce more particles. Most of these secondary particles are energetic enough to produce more particles in subsequent interactions with other nuclei. This showering process continues until the shower particles have too little energy to produce more particles.

Interspersed in a regular pattern with the calorimeter's dense metal are a large number of liquid argon ionization chambers through which the shower particles also pass. The charged shower particles ionize the liquid argon. This ionization then drifts in an electric field $\mathcal{E}$ toward the chamber electrodes inducing an electrical signal in an external circuit.

At the LHC design energy and luminosity the average rate of ordinary proton-proton collisions is about $1 \mathrm{GHz}$. In a typical $2 \mathrm{~mm}$ ionization chamber gap with a potential of $2000 \mathrm{~V}$ across the electrodes, the electrons drift out in about $434 \mathrm{~ns}$ [4] while the positive argon ions take of order $25 \mathrm{~ms}$. As a first approximation we consider the ionization rate as constant in time. The study of the performance of a liquid argon ionization chamber in this large, ap- 
proximately time-constant and spatially uniform ionization environment is the subject of this report.

We employ nine liquid argon ionization chambers, three at a time, in our study. Integrated into six of the nine chambers, two at a time, are beta sources distributed such that they ionize the argon approximately uniformly over a region of the chamber volume. The other three chambers act as controls. Each chamber is connected to an external circuit. Our observables are the currents through these external circuits. The parameters we vary are 1) the beta source activity (only two values), 2) the width of the chamber gap (only three values) and 3) the potential applied across the chamber gaps over an exceptionally wide range of values of both polarities at closely spaced intervals. The relatively simple geometry is amenable to straightforward predictions from analytic approximations and from more detailed and realistic simulations. We determine directly the threshold ionization rate above which space-charge effects degrade the performance of the liquid argon ionization chamber. This is the primary goal of this study. By comparison to predictions we are able to extract the positive argon ion mobility.

\section{Phenomenology}

High energy charged particles passing through liquid argon knock loose outer shell electrons from those argon atoms close to the particle track [5]. In each ionization an electron is separated from an argon atom leaving a positively charged argon ion. In the spectrum of ionization electrons, some (called delta rays) have sufficient kinetic energy to themselves create significant additional ionization. At each primary ionization process the high energy charged particle loses an amount of energy which is typically small compared to its kinetic energy.

Without an electric field, the ionization electron would likely recombine with its argon ion. But in the presence of an electric field the ionization electrons may escape and drift towards the anode while the argon ions drift towards the cathode. 
Several forms of recombination deplete the ionization electrons. We will divide these forms into two classes. In the first class the ionization electron may recombine with its argon ion. Or, in a dense cluster of ionization, as from a slowly moving delta ray, electrons may recombine with other ions produced by the same track or with other time-correlated tracks. We will call such processes "rate-independent recombination" (RIR). This form of recombination is independent of the rate of high energy charged particles producing tracks in the chamber and dominates the recombination processes at low rates where the tracks are widely separated in time. The wide separation makes it unlikely the ionization from one track overlaps with that from a non-time-correlated track. The so-called High Voltage Plateau Curve of current versus electric field, normalized to unity at asymptotic potential [6], can be interpreted as the probability that the ionization charges survive these RIR mechanisms.

For these RIR processes we will assume the electron and ion recombine so quickly that they do not drift far enough to contribute to a current in the electrical circuit attached to the electrodes [7].

The second class occurs when the rate of high energy charged particles is high enough that the bulk density of drifting electrons and positive charges can become significant. The probability that an ionization electron produced by one high energy charged particle combines with an ion produced by a different high energy charged particle becomes large. In such a "rate-dependent recombination" (RDR) process the probability of recombination depends on the rate of the high energy charged particles. Here the electron and ion may drift an appreciable distance before recombining, thus contributing to the current induced in the external circuit.

As we raise the ionization rate in a liquid argon ionization chamber the density of the slowly drifting positive argon ions builds up and distorts the electric field in the gap between the electrodes. But as long as the electric field is significant everywhere in the gap, the highly mobile electrons drift out of the gap at a rate which is high compared to the bulk recombination rate and, therefore, the RDR does not appreciably affect the current in the external 
circuit. But with further rate increases we eventually reach a threshold where the electric field near the anode is so distorted that it is almost completely screened, i.e. the electric field is infinitesimal. The density of electrons then also builds up in this same region to equal the density of the positive argon ions so the net charge density is zero. All ionization created in this region suffers the RDR so the only ionization contributing to the current in the external circuit is that created outside of this region of the chamber. As the ionization rate increases further, this screened region expands toward the cathode. The current in the external circuit is no longer directly proportional to the ionization rate. We say that the ionization chamber is in the space-charge limited regime. A full discussion can be found in reference [8].

We define $D_{i}$ as the ionization rate-density at finite $\mathcal{E}$-field. That is, it is the ionization rate at asymptotic $\mathcal{E}$-field minus the ionization lost to RIR processes at the working $\mathcal{E}$-field. In order for us to map out the transition into the spacecharge limited regime we would ideally vary the ionization rate by varying the activity of the beta source at fixed potential and measure the current density $J$. As we raise the source activity we would expect [8] the measured current density to rise in direct proportion until the ionization rate $D_{i}$ reaches the critical value $D_{c}$. Above this critical value the electron and argon ion charge densities build up to levels where the RDR becomes significant and the current density would then rise more slowly than the source activity.

But we have only two source activities, not a continuum, so we have developed a different approach. This approach is based on the observation that the critical ionization rate $D_{c}$ depends on the potential $V_{0}$ applied across the two electrodes of the liquid argon ionization chamber. Instead of varying $D_{i}$ (by varying the source activity) we, instead, vary $D_{c}$ (by varying $V_{0}$ ).

But in varying $V_{0}$ we not only change $D_{c}$ we also change $D_{i}$ because the RIR depends on $V_{0}$. However, by accurately measuring the HV plateau curve we can correct for the change in $D_{i}$ due to the RIR. So our strategy is the following: We first determine the HV plateau curve using the weaker of our two beta sources. We then use this HV plateau curve to separate the two classes of recombination 
processes which modify the current density from the stronger of the two beta sources. The RDR effect depends on the ionization rate and is significant only when the ionization chamber is driven into the space-charge limited regime.

Here we develop an analytic calculation to frame our further discussion and analysis. In order to derive simplified expressions certain approximations were necessary. We will identify our assumptions and their short-comings and overcome these short-comings with detailed numerical simulations described in section 7 .

The beta source with the weaker activity is numbered 1 and the source with the stronger activity is numbered 2 . The strong source has activity $A$ times larger than the activity of the weak source. At asymptotic potential (where the RIR and RDR can be ignored), the ionization rates in the liquid argon ionization gaps, here assumed to be homogeneous throughout, are $D_{0}$ and $A D_{0}$ respectively where $A$ is of order 20 . At finite potentials, the ionization rates are $D_{i 1}=D_{0} P\left(V_{0} / a\right)$ and $D_{i 2}=A D_{0} P\left(V_{0} / a\right)$ where $a$ is the gap width between the two electrodes and $P\left(V_{0} / a\right)$ is the HV plateau curve normalized to unity at asymptotic potential. Here we are assuming that the electric field in the ionization chamber is equal to the average value $V_{0} / a$. This requires an electrode structure approximating parallel plates and a negligible charge density between them. This latter assumption is a poor one when the charge densities in the ionization chamber are large enough, i.e. when the net charge in the gap becomes a major fraction of the charge on the electrodes. In this case the electric field in the chamber gap is not even approximately at the average value [8].

We will find it convenient to define the dimensionless parameters $r_{1} \equiv$ $D_{i 1} / D_{c}$ and $r_{2} \equiv D_{i 2} / D_{c}$. When $r>1$, the ionization chamber is in the space-charge limited regime. The critical ionization rate $D_{c}$ and the associated critical ionization current density $J_{c}$ are derived in ref. [8] and in other references cited therein:

$$
D_{c}=\frac{4 V_{0}^{2} \epsilon \mu_{+}}{a^{4}} \quad J_{c}=a D_{c}
$$


where $\epsilon$ is the permittivity for liquid argon $\left(\epsilon=1.51 \epsilon_{0}\right)$ and $\mu_{+}$is the positive argon ion mobility.

Again, using ref. [8] we write the net current densities (including the RDR) generated in our ionization chambers by the two source activities:

$$
\begin{aligned}
& J_{1}= \begin{cases}r_{1} J_{c} & \text { for } r_{1}<1 \\
r_{1}^{3 / 4} J_{c} & \text { for } r_{1}>1\end{cases} \\
& J_{2}= \begin{cases}r_{2} J_{c} & \text { for } r_{2}<1 \\
r_{2}^{3 / 4} J_{c} & \text { for } r_{2}>1\end{cases}
\end{aligned}
$$

For each source activity we can vary $r$ so that the ionization rate in the chamber is either in $(r>1.0)$ or out of $(r<1.0)$ the space-charge limited regime. We can vary $r$ by varying $D_{c}$. And we vary $D_{c}$ by varying $V_{0}$.

Let us now define the critical potential $V_{c}$ as the potential across the electrodes at which $D_{c}=D_{i}$, i.e. where $r=1$. For a given gap width there will be a different value of $V_{c}$ for each of the two different source activities. These two values are obtained by solution of the two transcendental equations:

$$
\frac{D_{0} a^{4}}{4 \epsilon \mu_{+}}=\frac{V_{c 1}^{2}}{P\left(V_{c 1} / a\right)} ; \quad \frac{A D_{0} a^{4}}{4 \epsilon \mu_{+}}=\frac{V_{c 2}^{2}}{P\left(V_{c 2} / a\right)}
$$

Note that $V_{c 2}>V_{c 1}$ and $r_{2}>r_{1}$ for the two different ionization chambers with the same $V_{0}$ and $a$. When $V_{0}>V_{c 2}$, then $r_{2}<1$ (and, of course $r_{1}<1$ ) so at a given $V_{0}$, we have

$$
J_{2} / J_{1}=r_{2} / r_{1}=D_{i 2} / D_{i 1} \equiv A
$$

As 4 we lower $V_{0}$ below $V_{c 2}$ but still above $V_{c 1}$, i.e. for $V_{c 1}<V_{0}<V_{c 2}$, gap 2 is in the space-charge limited regime but gap 1 is not and we get 


$$
\begin{aligned}
& J_{1}=a D_{0} P\left(V_{0} / a\right) \\
& J_{2}=A a D_{0} P\left(V_{0} / a\right)\left(\frac{V_{0}}{V_{c 2}}\right)^{1 / 2}\left(\frac{P\left(V_{c 2} / a\right)}{P\left(V_{0} / a\right)}\right)^{1 / 4} \\
& J_{2} / J_{1}=A\left(\frac{V_{0}}{V_{c 2}}\right)^{1 / 2}\left(\frac{P\left(V_{c 2} / a\right)}{P\left(V_{0} / a\right)}\right)^{1 / 4}
\end{aligned}
$$

The last factor is close to unity when the HV Plateau Curve is relatively flat between $V_{c 1}$ and $V_{c 2}$. And, even if not, the small value of the exponent makes this factor close to unity. So, as a further approximation, we can ignore this factor. Then we expect the ratio of current densities in the region $V_{c 1}<V_{0}<V_{c 2}$ to be approximately

$$
J_{2} / J_{1}=A\left(\frac{V_{0}}{V_{c 2}}\right)^{1 / 2}
$$

As we further lower $V_{0}$ below $V_{c 1}$ both gaps enter the space-charge limited regime and we get

$$
\begin{aligned}
& J_{1}=a D_{0} P\left(V_{0} / a\right)\left(\frac{V_{0}}{V_{c 1}}\right)^{1 / 2}\left(\frac{P\left(V_{c 1} / a\right)}{P\left(V_{0} / a\right)}\right)^{1 / 4} \\
& J_{2}=A^{3 / 4} J_{1} \\
& J_{2} / J_{1}=A^{3 / 4}
\end{aligned}
$$

So, as the potential $V_{0}$ across each of the pair of electrodes is varied from infinity down to zero, the ratio of current densities from the two beta source activities should range from a high value of $A$ down to the low value of $A^{3 / 4}$ with a transition region starting at $V_{c 2}$ and ending at $V_{c 1}$. In the ratio of the strong source current to the weak source current the variation due to the HV plateau curve largely cancels out. A measurement of $V_{c 2}$ and $V_{c 1}$ gives the ionization rate at which each liquid argon gap passes through the transition from normal operation into the space-charge limited regime. And because $V_{c 2}$ and $V_{c 1}$ depend on the argon ion mobility $\mu_{+}$we can extract this previously poorly known parameter from our data. 


\section{Beta Sources}

The betas which ionize the liquid argon originate from a Strontium-90 $\left({ }_{38}^{90} \mathrm{Sr}\right)$ source with half-life of 28.8 years. Strontium-90 emits a beta with a kinetic energy spectrum whose end-point is $0.546 \mathrm{MeV}$. The decay product is Yttrium$90\left({ }_{39}^{90} \mathrm{Y}\right)$, also a beta emitter with a kinetic energy spectrum whose end-point is $2.281 \mathrm{MeV}$ and half-life of 2.67 days. The decay product of Yttrium-90 is stable Zirconium-90 $\left({ }_{40}^{90} \mathrm{Zr}\right)$. The few gammas are produced primarily via Bremsstrahlung. Beta spectra can be found in ref. [9]. It is traditional to quote only the activity of the Strontium but, within weeks of production, the Yttrium has accumulated to an activity almost equal to that of Strontium so the actual activity is nearly twice the quoted value.

\section{Apparatus}

Each liquid argon ionization chamber structure consists of two electrodes. One electrode is a solid copper rod of diameter of $4.712 \mathrm{~mm}$ for the strong source and $4.702 \mathrm{~mm}$ for the weak source. In the following we will refer to the dimensions for the strong source only but we should remember that the rod diameter for the weak source is slightly smaller, making all gaps for the weak source slightly larger than the gaps for the corresponding strong source. Into one end of this rod a co-axial cavity (diameter of $4.212 \mathrm{~mm}$ ) has been hollowed out to a depth parallel to the axis of about $14 \mathrm{~mm}$. A Strontium-90 beta source was uniformly deposited onto one side of a $60 \mu \mathrm{m}$ thick by $10 \mathrm{~mm}$ long copper foil. The foil was then rolled into a cylinder, with the source material on the outside surface, and inserted into the cavity such that the restoring force of the foil caused it to hug the cavity wall. A solid copper rod of diameter $4.712 \mathrm{~mm}$ with a concentric shaft of diameter $3.0 \mathrm{~mm}$ and length $19 \mathrm{~mm}$ extending from one end, threaded at the end, was screwed into a threaded hole at the far end of the cavity and was then laser welded to the open end of the cavity, thus sealing the cavity with the source inside. From the outside the resulting assembly looks like one $150 \mathrm{~mm}$ long solid copper rod. 


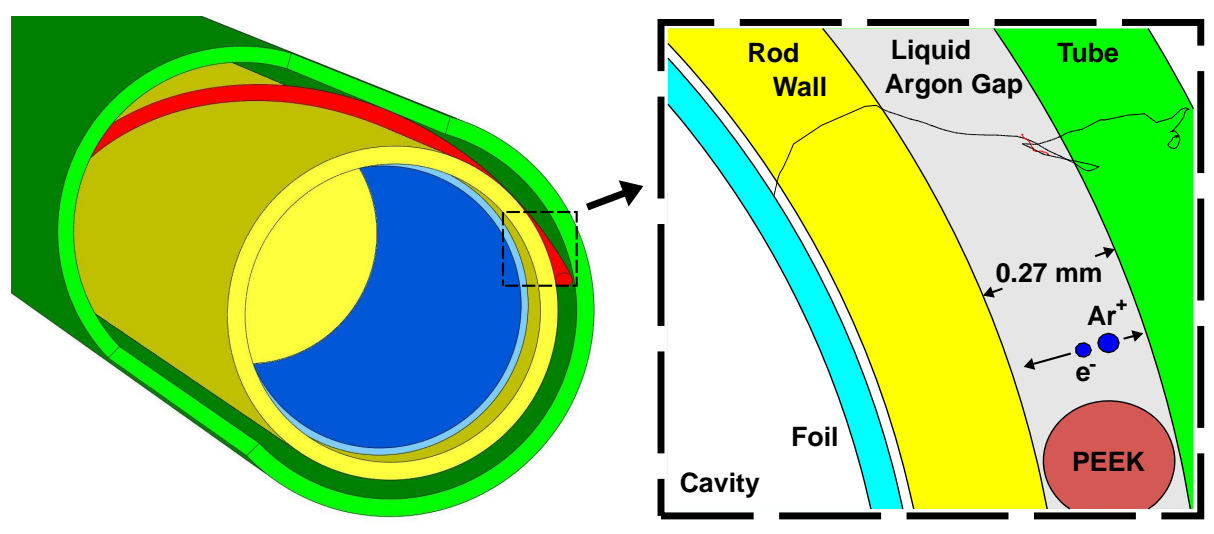

Figure 1: Liquid argon ionization chamber with Strontium-90 beta source. The perspective drawing on the left shows a cut-away of the two electrodes: a copper rod (yellow) within a thin-walled copper tube (green). The rod is held in place within the tube by an insulating PEEK fiber (red) wound helically around the rod. The gap between the rod and tube fills with liquid argon forming the ionization chamber. A power supply (not shown) provides a potential difference between the rod and tube. The solid copper rod has a concentric cavity machined out to a depth, along the axis of the rod, of $14 \mathrm{~mm}$. Inserted into this cavity is a rolled copper foil (blue) of $10 \mathrm{~mm}$ length, coated on the outer surface with the Strontium-90 beta source. The drawing on the right shows a cross-sectional close-up of this structure. Overlaid is the track of one simulated beta, from the Yttrium decay product of a Strontium-90 decay, leaving the source on the surface of the foil with kinetic energy of $1.48 \mathrm{MeV}$ and passing through the liquid argon gap. An energetic delta ray, produced by this beta in the liquid argon, is represented by the red track.

Three different types of tube were used for the other electrode. The first type is a stainless steel tube with inner diameter of $4.990 \mathrm{~mm}$ and outer diameter of $6.350 \mathrm{~mm}$. In this case a $102 \mu \mathrm{m}$ PEEK fiber, helically wound around the rod, holds the rod concentric within the $150 \mathrm{~mm}$ long tube leaving a gap of $138 \mu \mathrm{m}$ between the electrodes. The second type is a copper tube with inner diameter of $5.250 \mathrm{~mm}$ and outer diameter of $5.753 \mathrm{~mm}$. Here a $250 \mu \mathrm{m}$ PEEK fiber, helically wound around the rod, holds the rod concentric within the $150 \mathrm{~mm}$ long tube leaving a gap of $268 \mu \mathrm{m}$ between the electrodes. The third type is a copper tube with inner diameter of $6.51 \mathrm{~mm}$ and outer diameter of $7.01 \mathrm{~mm}$. In this case the helically wound polystyrene fiber has diameter of $835 \mu \mathrm{m}$ and 
the gap is $898 \mu \mathrm{m}$. When in the cryostat, liquid argon fills the gap between the two electrodes. The second of these ionization chamber structures, without the rod with the shaft, is shown in Fig. 1. In liquid argon the capacitance of the chambers with a gap of $138(268,898) \mu \mathrm{m}$ is about $214(117,39.0) \mathrm{pF}$.

Two such rod assemblies were produced in October of 2007 with source activities specified to be $2 \mathrm{mCi}$ (weak source) and $50 \mathrm{mCi}$ (strong source). A third rod assembly with no beta source $(0 \mathrm{mCi})$ was used as a control. Each of the three rod assemblies was inserted into three identical tubes which were one of the three tube types. These three physically identical chambers, differing only in source activity, were inserted into three of four holes drilled near the center of a solid copper cube $150 \mathrm{~mm}$ on a side. (The copper cube was designed to minimize personnel radiation exposure.) The holes formed a $2 \times 2$ square array with center-to-center nearest-neighbor separation of $12 \mathrm{~mm}$. The strong source chamber was equidistant from the $0 \mathrm{mCi}$ and from the weak source chambers so that the $0 \mathrm{mCi}$ chamber could measure the radiation from the strong source leaking through to the weak source chamber.

The copper cube with the ionization chambers was mounted in a liquid argon cryostat which had been cleaned with alcohol. After closing the cryostat the interior was evacuated and then filled with argon gas which had evaporated from a liquid argon dewar and had then passed through a getter [10]. This evacuation and filling process was repeated several times with modest heat applied to bake out moisture. After the final filling, liquid nitrogen was flowed through coils near the top of the cryostat. The argon gas pressure was adjusted to about 0.1 bar above the room pressure of about $0.93 \pm 0.02$ bar and argon condensed on the coils, eventually filling the cryostat to the top of the copper cube. A tube extending to the bottom of the cryostat and penetrating the top of the cryostat was connected to two oxygen analyzers in parallel [11]. When the valves to the oxygen analyzers were opened, liquid argon rose in this tube to a level a little above the level in the cryostat where it evaporated. The gas flow rate was slow enough that the tube temperature remained above the ambient dew point at the penetration. The oxygen contamination was below $0.25 \mathrm{ppm}$. Argon gas 


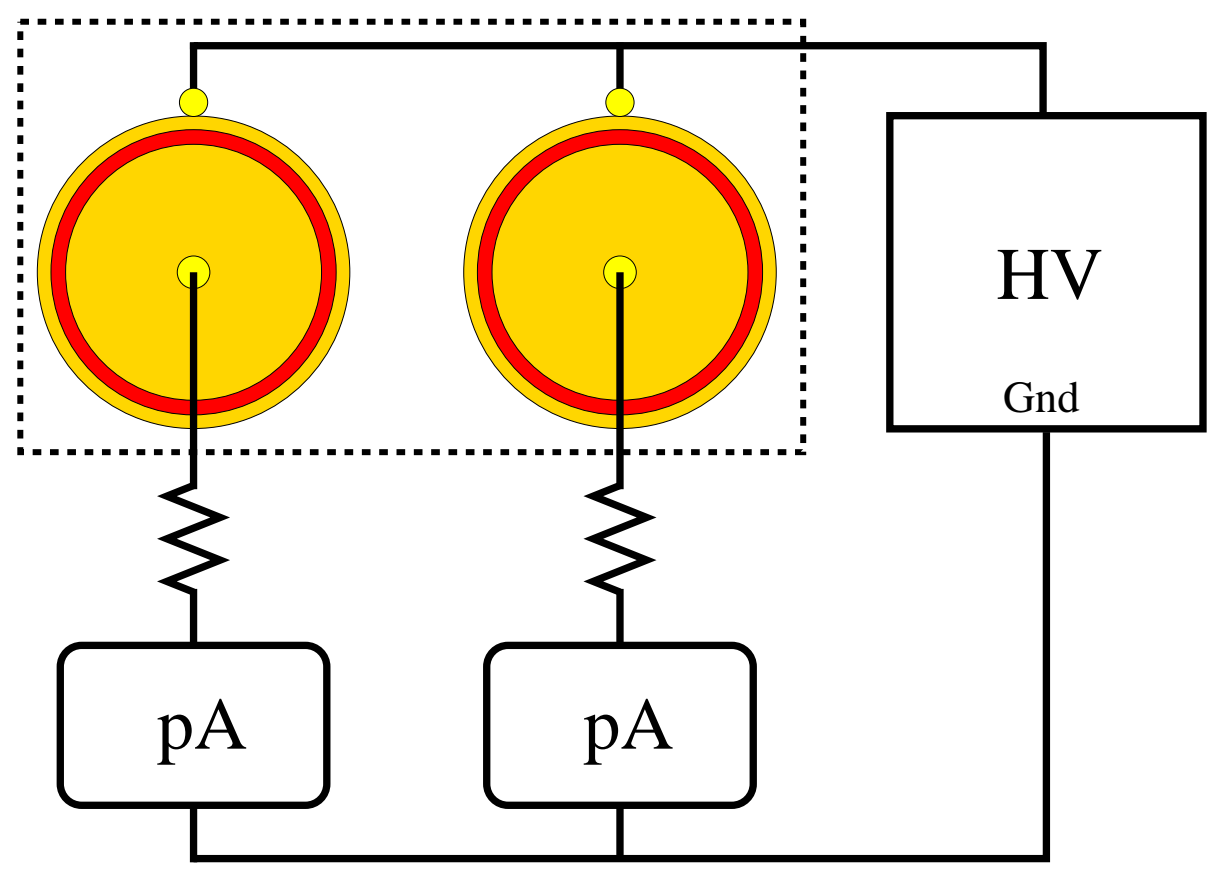

Figure 2: Schematic current readout circuit. Shown at the signal connection end are two ionization chambers with copper parts in yellow and liquid argon in red. HV is the high voltage power supply and each pA is a picoAmmeter. The tubes are at high voltage, either positive or negative, while the picoAmmeters are connected to the ground of the supply.

from the argon dewar was slowly flowed into the cryostat through the getter to make up for the loss through the oxygen analyzers. The liquid nitrogen flow rate was controlled by a nitrogen-gas-powered pressure regulator and valve located at the output of the cooling coils after a long run through a heated copper tube. The nitrogen gas at the regulator valve was warm enough that no condensation and freezing would affect the valve. Excess nitrogen gas was then routed to the signal feedthroughs to prevent condensation of moisture from the room air, thereby minimizing leakage currents. A separate circuit monitored the negligible leakage currents.

The liquid argon temperature was measured with three temperature sensors [12] and the absolute pressure of the argon gas above the liquid surface was measured with a mechanical gauge [13]. 
The tubes of the three ionization chambers in use at any one time were wired together to a $\mathrm{HV}$ feedthrough. This feedthrough was then connected to one of four HV power supplies $[14,15]$. Each of the rods of the three ionization chambers was connected to its respective signal feedthrough. Only two of these feedthroughs were connected to the two picoAmmeters [16] for any one run. The simplified wiring diagram in Fig. 2 shows these connections. Each circuit has a protection resistor in series with the picoAmmeter, $4 \mathrm{M} \Omega$ for the strong source chamber and $10 \mathrm{M} \Omega$ for the weak source chamber. Because of the small currents in this study, the potential drops across these resistors required only a very small correction.

\section{Data Taking}

Data acquisition was managed automatically by a PC running LabVIEW [17]. Once a run was started the LabVIEW program cycled through a series of readings. In each cycle it read the two picoAmmeters, a monitor of the HV supply potential, and the cryostat pressure transducer. After each cycle it recorded these readings to a disk file, along with the time since the beginning of the run and the value of the potential it sent to the HV supply. Each cycle took approximately 0.5 seconds. At one time during the run the $\mathrm{PC}$ read out two temperature probes located in the liquid argon at a depth close to the ionization chambers. These were recorded at the end of the run. The date and time (to the nearest second) of the start of the run was encoded into the raw data file name.

At the beginning of each run the LabVIEW program read in a command file which contained the values of potential to which the HV supply should be set. For each value of potential there were 40 cycles of readings (taking approximately 20 seconds). The file started with 90 potential values of zero so there were 3600 cycles of readings with the HV supply set to zero. Then there was one non-zero potential value (40 cycles) followed by another 30 to 40 values of zero. We call these non-zero potentials "steps" because when the LabVIEW 
program progressed to such a value it stepped-up the potential from the nominal zero value to the potential read from the command file. This change in potential usually occurred between two cycles of readings but sometimes there was a cycle with readings somewhere between the readings at zero potential and the readings at the requested potential. At the end of the step, i.e. after 40 cycles of readings, the LabVIEW program set the HV supply potential back to zero. But it took many cycles for the current readings to return to their values before the step.

The range of HV potentials in a run was limited by the HV power supply. To cover the full range of potentials in this study we used four HV power supplies, two of which had a manual switch to select one of two ranges. Half of these HV power supplies provided positive potentials and the other half provided negative potentials. We covered the full range of potentials in eight runs, usually taking eight days.

For the primary data runs, the two picoAmmeters read out the strong source and the weak source ionization chambers. But we interspersed background eight-run sequences reading out the $0 \mathrm{mCi}$ ionization chamber and what we called the "leakage" channel. This channel had connections from the HV supply and picoAmmeter to the cryostat feedthrough. But nothing was connected to this feedthrough on the inside of the cryostat. The purpose was to measure any residual leakage currents.

The cryostat was filled with liquid argon and we started taking data with the $268 \mu \mathrm{m}$ ionization chambers on 11 February 2009. We took data continuously until 18 December 2009 at which time we warmed up the cryostat. We filled the cryostat again and started taking data with the $898 \mu \mathrm{m}$ ionization chambers on 19 March 2010. We took data continuously until 2 August 2010 and again warmed up the cryostat. We started taking data with the $138 \mu \mathrm{m}$ ionization chambers on 10 November 2010 and ended on 17 December 2011.

We raised the potential on the various ionization chambers until the argon broke down, drawing excessive current which then tripped the power supply. For production running we backed off a bit from the trip point so as to avoid excessive trips. The electric fields we achieved are much larger than is often 


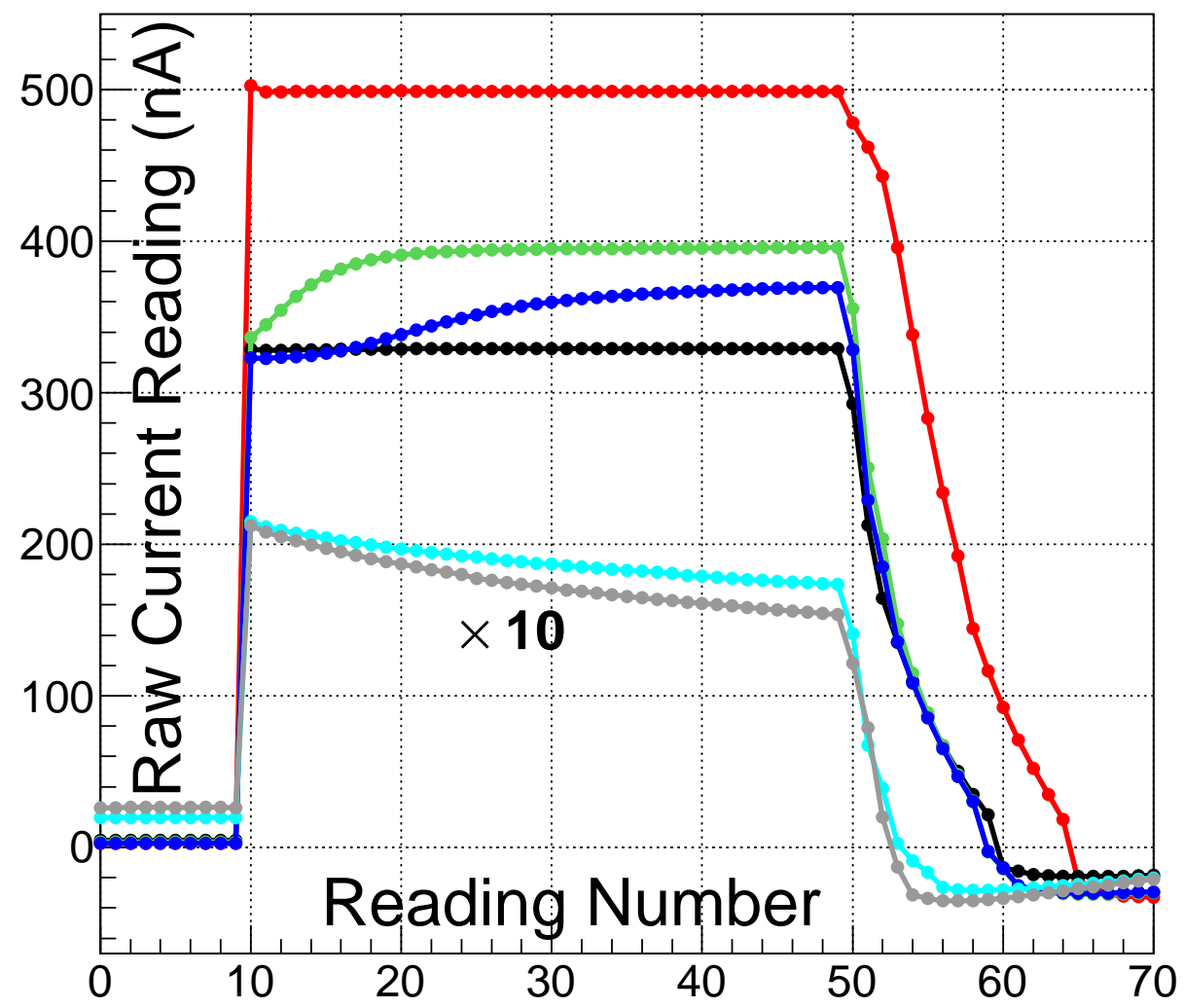

Figure 3: Raw current readings from the $268 \mu \mathrm{m}$ strong source gap for six good steps at three different potentials (from top to bottom, red $-260 \mathrm{~V}$, green $-60 \mathrm{~V}$, dark blue $-60 \mathrm{~V}$, black - $60 \mathrm{~V}$, cyan $-1.0 \mathrm{~V}$, and gray - $1.0 \mathrm{~V}$ ) superimposed in time. The current readings for the two steps at $1.0 \mathrm{~V}$ have been multiplied by 10 . The potential was turned on between reading numbers 9 and 10 and turned off between reading numbers 49 and 50 .

possible in planar electrode structures with the same gap. We have never observed a breakdown which didn't clear after lowering the potential. That is, we have never developed a short across the gap.

\section{Data Reduction}

We examined plots of the raw data starting from current readings just before a potential step to just after that potential step to spot anomalies in the data.

Fig. 3 shows a plot of two typical ideal steps in red and black. Most steps look 

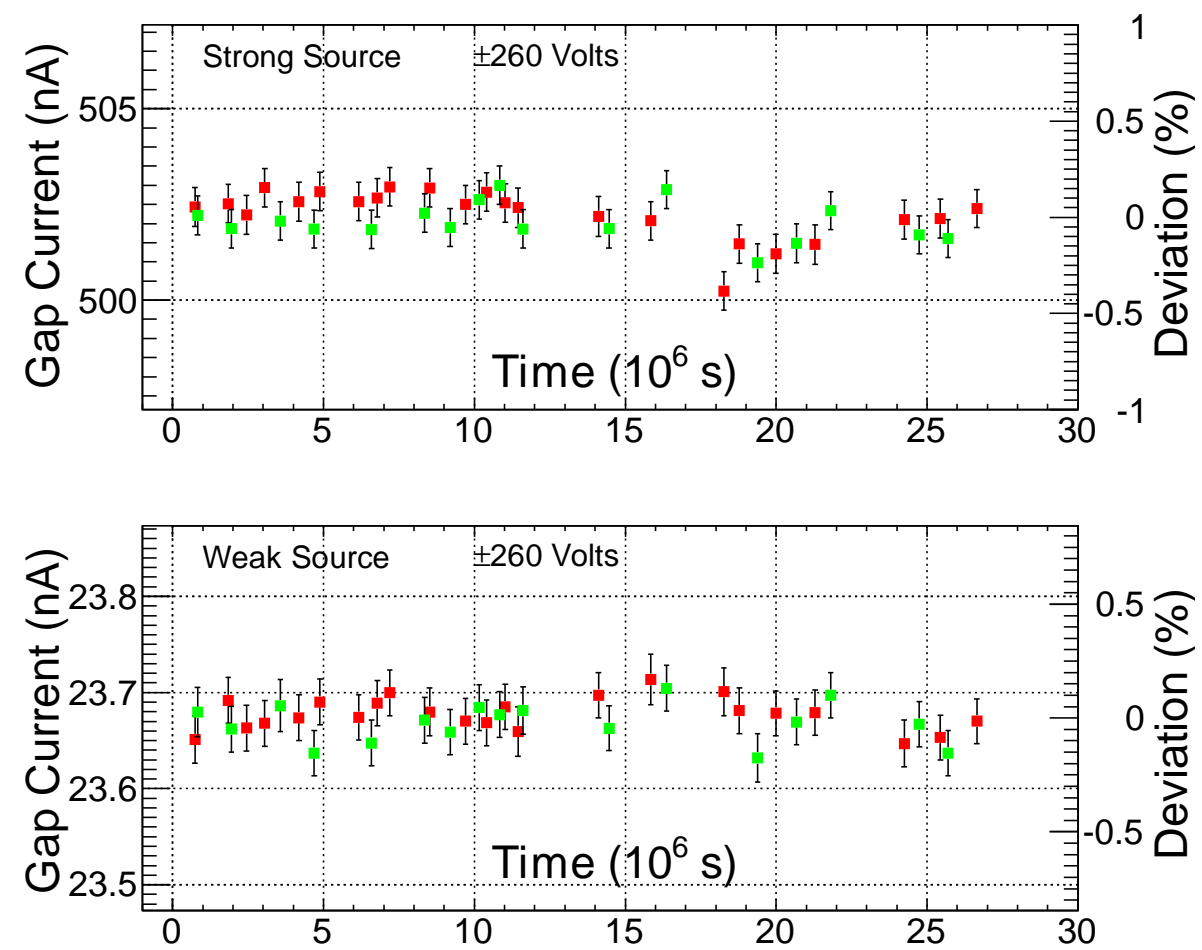

Figure 4: Step currents in the $268 \mu \mathrm{m}$ ionization chambers at the operating potential of 260 $\mathrm{V}$ as a function of the time of the run, after correction for the decay of the beta sources. Red (green) data points are at positive (negative) polarity. The error bars are the systematic uncertainties described in the text. This potential corresponds to the standard electric field of approximately $1 \mathrm{kV} / \mathrm{mm}$ used by many liquid argon calorimeters.

like these. The rest of the steps in Fig. 3 are classified as good steps but are a challenge to interpret. For the ideal case in red, note that current reading 10 is slightly higher than the succeeding readings. This is due to the excess current required to charge the chamber electrodes. The charging time constant was of order $30 \mathrm{~ms}$ so we see only the tail of this charging current on top of the ionization current. Ranges of good steps were determined for all runs. Most often the range for a run included all of the steps in that run.

The HV supply would automatically reset within seconds of a trip so it was sometimes possible to classify a step as good even if a trip occurred. But usually steps with a trip were classified as bad. 


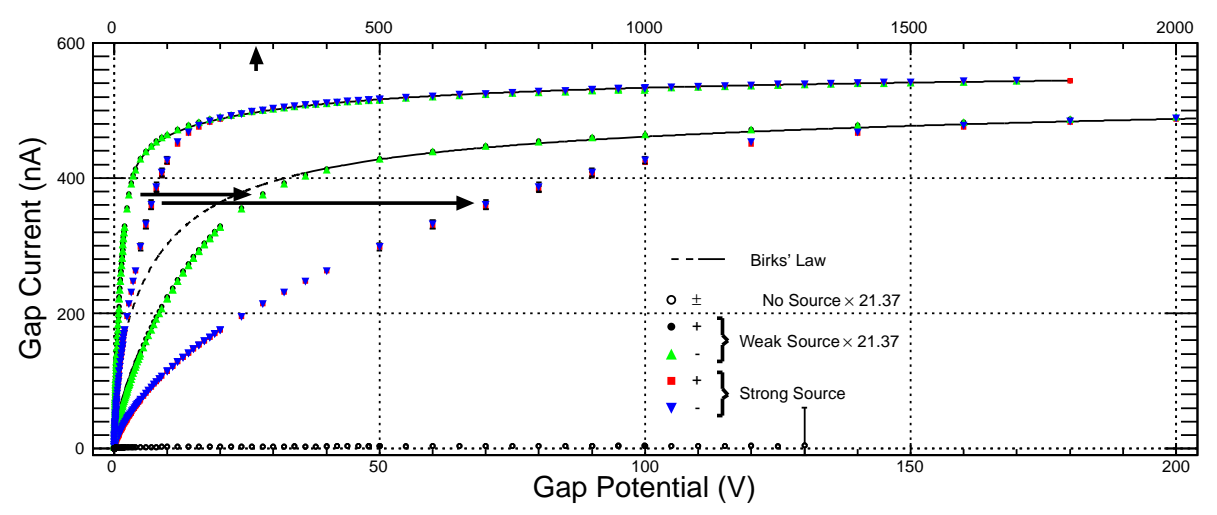

Figure 5: Step currents from the ionization chambers with $268 \mu \mathrm{m}$ gaps versus the potential applied across the electrodes of the ionization chambers. Both polarities are shown. For data taken with the negative HV supply the currents and potentials have been multiplied by -1 . For potentials at and below $200 \mathrm{~V}$ the data points are plotted twice, once using the potential axis at the bottom of the plot and once using the potential axis at the top of the plot. The two horizontal arrows each connect two of many identical data points, the one on the left plotted against the top axis and the one on the right plotted against the bottom axis. For potentials above $200 \mathrm{~V}$ data points are plotted only once using the axis at the top. The step currents from the weak source ionization chamber are multiplied by 21.37 to show how the step currents agree in shape at higher potentials. The step currents from the $0 \mathrm{mCi}$ ionization chamber, multiplied by 21.37, are also shown. A solid curve shows the HV plateau curve of Eq. 11 in the region of potential where the data was fit. This parameterization, extrapolated to lower potentials, is shown as a dashed line. A vertical arrow on the top axis shows the nominal operating point for many liquid argon calorimeters.

After rejecting such bad steps (and occasionally whole bad runs) the raw data was analyzed to extract a summary current which we call either the step current or the gap current for each potential step from the 40 current readings on the step and from 10 current readings just before the step. At the lower potentials the current readings during the step were not constant. Example steps at 1.0 $\mathrm{V}$ are shown in Fig. 3 in cyan and gray, multiplied by a factor 10 . We assume a thin, insulating layer builds up on the electrodes perhaps from ionized impurities in the liquid argon. This thin layer has a very large capacitance and breaks down at low potentials somewhat like a reverse-biased zener diode. Our data-taking procedure was adapted to avoid complications from this presumed effect. For 
the runs covering a range of low potentials we fit the individual current readings on the step with a quadratic polynomial and extrapolate back to the beginning of the step where we presume no potential has yet built up across the thin layer. Therefore at that time the applied potential is across the liquid argon gap and none is yet across the layer. As the potential across the layer slowly builds up, the potential across the liquid argon gap drops, causing the current to drop as well. In this fit we excluded the first four current readings after the beginning of the step in order to avoid the distortion due to the charging of the chamber capacitance. (The layer capacitance is many orders of magnitude larger than the chamber capacitance. So the potential across the liquid argon gap is established in tens of milliseconds while the potential across the layers takes many seconds to build up.) We also excluded current readings after reading number 30 (i.e. 20 after the beginning of the step) because we didn't have a fit function which would reasonably represent all shapes. We assign a systematic uncertainty to the extrapolated step current due to this procedure of one third of the difference in the current extrapolated to the beginning of the step and the current averaged over the last 20 current readings in the step. A more detailed description of this layer effect and our method for avoiding its consequences can be found in Ref. [18]. The potential step at $1.0 \mathrm{~V}$ in cyan was taken about 7 months before the one in gray. While they both extrapolate to approximately the same current at the time of reading 10, the later potential step falls faster with time suggesting a smaller capacitance, a higher breakdown potential, and therefore a thicker layer. In special runs we recorded steps at these low potentials as long as one hour and were unable to determine an asymptotic current. An asymptotic current would indicate the breakdown potential of the layer.

For steps at potentials just below the critical potential $V_{c}$ we observe a variety of pathologies. For some runs the raw current readings, such as the black current readings at $60 \mathrm{~V}$ in Fig. 3, are constant as they are for the $260 \mathrm{~V}$ step (red) but for other apparently identical runs the raw current readings rise over a period of seconds and then level off. Examples are the two $60 \mathrm{~V}$ steps (green and dark blue) in Fig. 3. We have been unable to correlate this behavior 


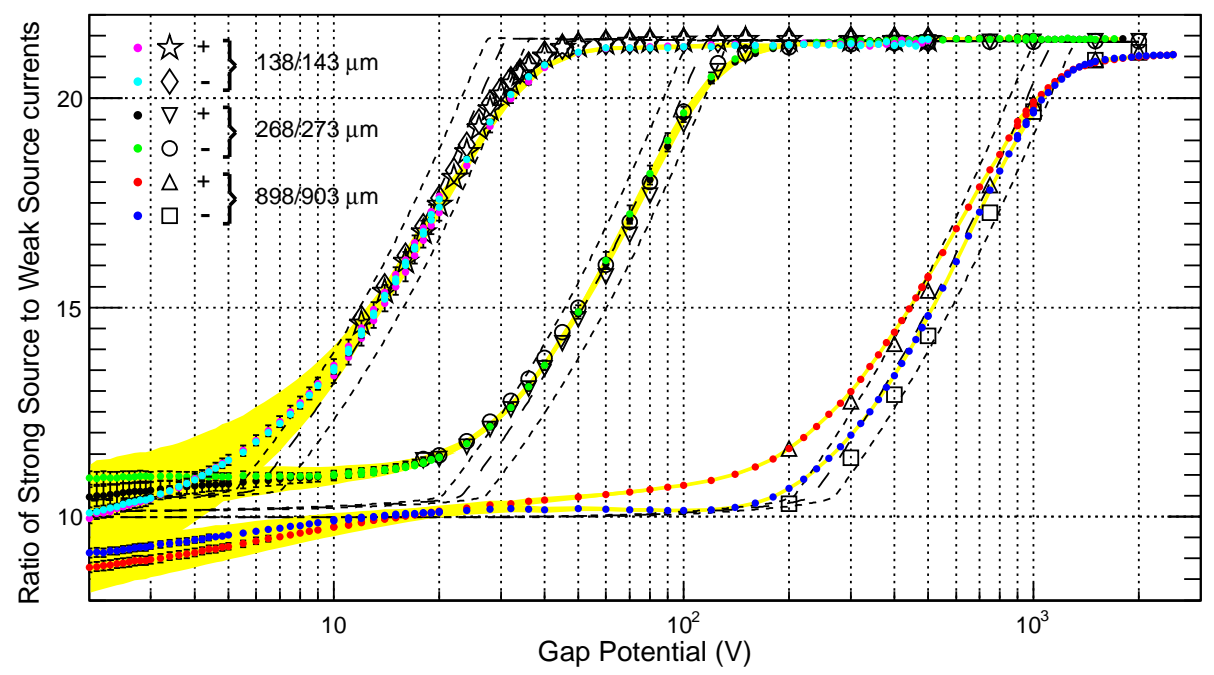

Figure 6: Ratio of the strong source chamber step currents to the weak source chamber step currents as a function of the potential across the ionization chamber for, from left to right, the $138 \mu \mathrm{m}$ gap, the $268 \mu \mathrm{m}$ gap, and the $898 \mu \mathrm{m}$ gap. Data for the two polarities of potential and the three gaps are shown as small, solid circles. In many cases the data points for the negative polarity overlap the data points for the positive polarity, obscuring them. The yellow bands indicate the overall systematic uncertainty in the data. Simulation data points are represented by open markers.

with any other observable and we have no explanation for it. We arbitrarily extract step currents for these steps and assign a systematic uncertainty via the same procedure as described above.

At the higher potentials the current readings on the step after the first few readings were constant throughout the rest of the step. If arcing occurred at the highest potentials it typically happened early in the step and then the gap rapidly recovered allowing constant current readings from there to the end of the step. To avoid discarding too much of the highest potential data we averaged the last 20 current readings and used this for the summary step current in place of the extrapolation to the beginning of the step.

The pedestal current before the step is not exactly zero. This is due in part to the HV power supplies which don't put out exactly zero potential. It is also due to some residual potential which we presume to be across the putative 
layer. The potential applied at the step is on top of this residual potential so we use the pedestal current to estimate this residual potential across the liquid argon gap and correct the step current using the slope of the step current versus potential curve at the applied potential. We assign a systematic uncertainty of half the magnitude of the correction.

There is a small potential drop across the protection resistors (see Fig. 2). Rather than correct the potential we instead correct the step current, again using the slope of the step current versus potential data. We do this so that we can directly compare step currents from the different chambers at the same potential data-point by data-point. No uncertainty is attributed to this correction.

The individual systematic uncertainties, along with an additional $0.1 \%$, are added in quadrature to obtain the overall systematic uncertainty for each step current. The systematic uncertainty is negligible for potentials above $V_{c 2}$, i.e. in the range of normal operation, and is large in the space-charge limited regime.

Summary data for each step in a run was written to an intermediate file. These files were read by a program which corrected the step currents for the small decay in activity parameterized by the half-life of Strontium-90. For a given potential, these corrected step currents were plotted as a function of the time of the run. This allowed us to inspect the stability of the data with time. An example is shown in Fig. 4. The rms fluctuations in the step currents shown in this figure are about $0.1 \%$ with no apparent trend. The data suggests that the stability of the liquid argon ionization chamber at high ionization rates is at least as good as our ability to measure it.

The rms deviation from the average of the multiple step currents at the same potential is used as the statistical uncertainty. While this uncertainty is quite small for potentials such as was chosen for the data shown in Fig. 4, for potentials just below $V_{c}$ the rms deviation is much larger. This is likely related to the pathologies seen in the steps at $60 \mathrm{~V}$ shown in Fig. 3. We interpret this as an instability of unknown origin.

Before plotting, the weak source step currents were corrected for the radiation leaking into the ionization chamber from the nearby strong source chamber. 
This is done by use of the step currents measured in the $0 \mathrm{mCi}$ chamber which comes only from radiation from the equally nearby strong source chamber. This was important because the spectrum of betas and gammas penetrating a part of the copper cube and reaching the gap of the weak source chamber is different from the spectrum coming directly from the foil. In principle the Plateau Curve for a different spectrum will have a different shape. This is a very small but not quite negligible correction to the weak source step currents.

Fig. 5 shows the corrected step currents as a function of the potential across the gaps of the $0.268 \mu \mathrm{m}$ chambers. The step currents from the weak source chambers have been multiplied by $A=21.37$ to easily see that the data points overlap at the higher potentials. This overlap is expected for RIR processes. Moving to lower potentials we see the two sets of data points diverge. This is a manifestation of the onset of space-charge effects, i.e. RDR effects, in the strong source gap which we will analyze quantitatively. Moving to even lower potentials we see the weak source currents diverge from the HV plateau curve. At these low potentials the weak source gap is also in the space-charge limited regime.

The step currents from the $0 \mathrm{mCi}$ (no) source, multiplied by $A=21.37$, are shown to give a feeling for the correction (already made) to the weak source step currents. The leakage currents (not shown) are everywhere smaller than the $0 \mathrm{mCi}$ currents, roughly proportional to the potential with inverse slope of approximately $30 \mathrm{~T} \Omega$.

A plot of the ratios of the strong source step currents to the weak source step currents as a function of the potential applied to the ionization chambers is shown for the three chamber gaps in Fig. 6. At the highest potentials the ratios for the $138 \mu \mathrm{m}$ gap and the $268 \mu \mathrm{m}$ gap are constant at $A=21.37$. This confirms with some precision that the HV plateau curve is rate independent, i.e. it is an RIR effect. For the $898 \mu \mathrm{m}$ gap the ratios appear to be approaching $A=21.37$. Moving to lower potentials we approach a critical value $V_{c 2}$ (different for each of the three gap widths) where the ratios begin to fall rapidly. Continuing to lower potentials, the ratios approach a lower constant as the potential approaches 
a second critical value $V_{c 1}$ (also different for each of the three gap widths). We interpret the ratio data below $V_{c 2}$ as a manifestation of the strong source chamber entering the space charge limited regime. Below $V_{c 1}$ both the strong source and the weak source chambers are in the space charge limited regime.

Also shown in Fig. 6 are the analytic approximations in equations 5, 6, and 8 with the positive argon ion mobility $\mu_{+}$set to $0.08 \mathrm{~mm}^{2} / \mathrm{Vs}$. The neighbor lines show the result of increasing and decreasing $\mu_{+}$by $0.02 \mathrm{~mm}^{2} /$ Vs. Open symbols show the results of a detailed simulation which will be described in the next section.

\section{Simulations}

\subsection{Energy deposit}

The EGSnrc Monte Carlo simulation code for electron and photon transport [19] was used to predict the energy deposited in the liquid argon gap by the betas. The origin of each beta was chosen via a uniform random distribution somewhere on the outer surface of the foil. The initial direction was also chosen via a uniform random distribution within a solid angle of $4 \pi$. The initial kinetic energy of each beta was chosen from a random distribution which represents the beta spectra of the combined Strontium-90 and Yttrium-90 in equilibrium [9]. Each beta was then tracked, along with all produced delta rays, gammas, and the occasional positron, until the kinetic energy fell below the threshold of 30 $\mathrm{keV}$. Steps along each track in the liquid argon were limited to a maximum of $1 \mu \mathrm{m}$ in length. All energy deposits were tallied.

In a production simulation we generated $n_{\beta}=10000000$ betas. Of these only $m_{\beta}=806116(810279,821012)$ deposit any energy in the $138(268$, 898) $\mu \mathrm{m}$ liquid argon gap. The others either head off in the wrong direction or range out before reaching the gap. The total energy deposited in the $138(268,898) \mu \mathrm{m}$ liquid argon gap by all of the betas in the simulation is $E_{\text {Tot }}=72425(131271,337442) \mathrm{MeV}$. For a source activity of $A_{\beta}=50 \mathrm{mCi}$ 

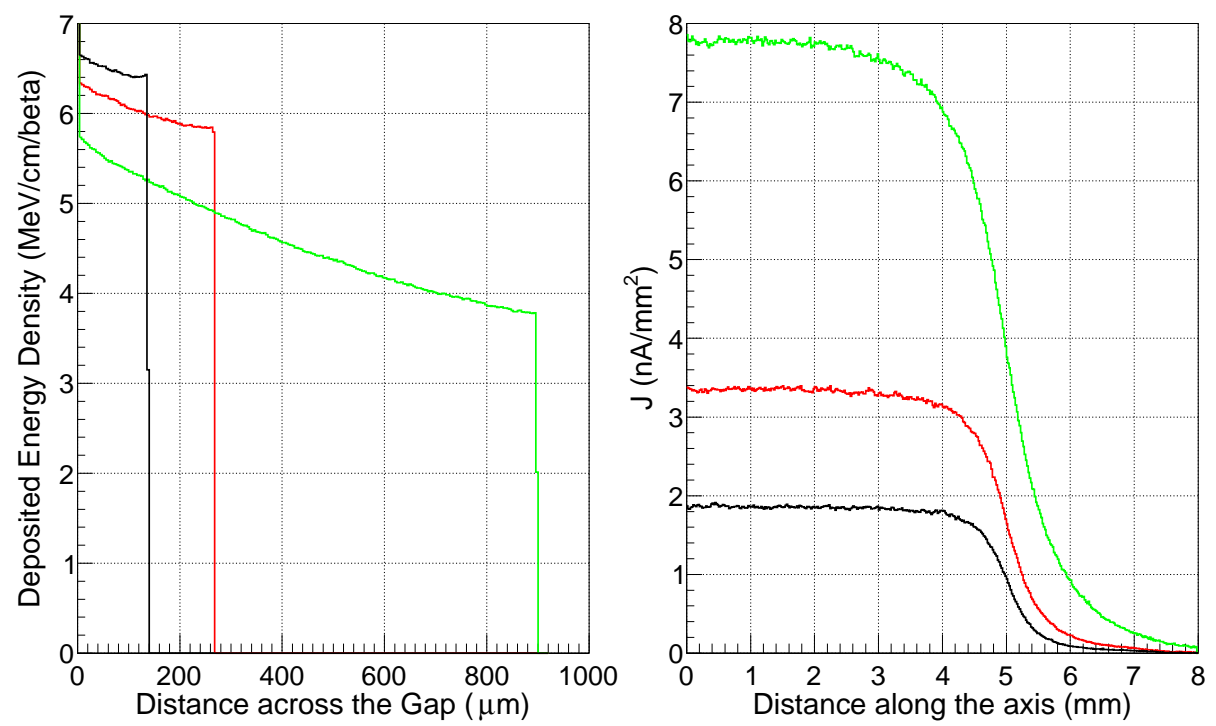

Figure 7: The plot on the left shows the average energy density deposited in the liquid argon gap as a function of distance across the gap in $1 \mu \mathrm{m}$ bins for the 10,000,000 generated betas. The Black (Red, Green) histogram is for the ionization chamber with the $138(268,898) \mu \mathrm{m}$ gap. The source is closest to the surface of the rod which, in the plot, is where the abscissa is zero. The plot on the right shows the simulated current density $J$ at the center of the gap for a strong source activity as described in the text at $\mathcal{E}_{\mathrm{T}}=22 \mathrm{kV} / \mathrm{cm}$, neglecting the RDR, as a function of $x$, the coordinate along the chamber symmetry axis. The Strontium-90 beta source extends from $-5<x<+5 \mathrm{~mm}$. Because of symmetry about $x=0$ we show only half of the relevant range. At $x= \pm 5.0 \mathrm{~mm}$ the current density has fallen to half the value in the uniform region. The same color code as on the left is used here.

the simulations predict the rate of betas entering the $138(268,898) \mu \mathrm{m}$ liquid argon gap is about $f_{\mathrm{g}}=298(300,304) \mathrm{MHz}$.

We convert the energy deposited in the liquid argon to ionization charge as follows: Measurements of the $976 \mathrm{keV}$ monochromatic kinetic energy conversion electron from ${ }^{207} \mathrm{Bi}$, ranging out in liquid argon, show the average deposited energy required to create one electron-ion pair as $W\left(\mathcal{E}_{\mathrm{T}}\right)=23.6 \pm 0.3 \mathrm{eV}$ at an electric field of $\mathcal{E}_{\mathrm{T}}=22 \mathrm{kV} / \mathrm{cm}$ [20]. Using the density of liquid argon $\left(1.396 \mathrm{~g} / \mathrm{cm}^{3}\right)$ and the ionization energy loss of minimum ionizing betas in the liquid $\left(1.349 \mathrm{MeV} /\left(\mathrm{g} / \mathrm{cm}^{2}\right)\right)[21]$ we find the average number of ionizations is 
about 8000 per mm of track length at $\mathcal{E}=\mathcal{E}_{\mathrm{T}}$. Considering that the kinetic energy spectrum of betas in the liquid argon from our Strontium-90 sources is softer than the Bismuth-207 betas but that some fraction of our Sr-90 betas have higher kinetic energy and pass through the liquid argon, ranging out in the copper tube, we guess that the RIR effects are about the same. We therefore use this same conversion factor $W\left(\mathcal{E}_{\mathrm{T}}\right)$. We use the High Voltage Plateau Curve to apply this conversion at other values of the electric field. That is, we normalize the High Voltage Plateau Curve to unity at $\mathcal{E}=\mathcal{E}_{\mathrm{T}}$ and interpret the resulting curve as the average number of electron-ion pairs which are created whenever the beta loses $23.6 \mathrm{eV}$ of kinetic energy and which survive RIR processes.

Using $e=1.602 \times 10^{-19} \mathrm{C}, W\left(\mathcal{E}_{\mathrm{T}}\right)=23.6 \mathrm{eV}$, and $1 \mathrm{Ci}=3.7 \times 10^{10} \mathrm{~Bq}$ we obtain the current at $\mathcal{E}_{\mathrm{T}}=22 \mathrm{kV} / \mathrm{cm}$ of

$$
I_{0}=\frac{e E_{\mathrm{Tot}}}{W\left(\mathcal{E}_{\mathrm{T}}\right)} \frac{2 A_{\beta}}{n_{\beta}}=181(326,815) \mathrm{nA}
$$

for a $50 \mathrm{mCi}$ source. With the $138(268,898) \mu \mathrm{m}$ ionization chamber, the measured current for the strong source, interpolated to $22 \mathrm{kV} / \mathrm{cm}$ or 304 (590, 1976) $\mathrm{V}$ and then correcting the $898 \mu \mathrm{m}$ data for RDR effects, is $284(520,1325) \mathrm{nA}$. So at the time of our measurements the activity of the strong source was actually $78(80,81) \mathrm{mCi}$. Both the strong source and the weak source were produced in 2007 and the activity determined by the manufacturer in November 2007 just before delivery. Extrapolating from the time of measurement to the production time we estimate the production activity of the strong source to be 86 $\mathrm{mCi}$. Using the ratio of currents at high electric fields we similarly estimate the production activity of the weak source to be $4.0 \mathrm{mCi}$. These are substantially higher than we requested.

The analytic calculations described in section 2 assume the energy deposition is uniform over the full volume between parallel plate electrodes. Fig. 7 shows the radial (left) and axial (right) dependence of the energy deposition in the cylindrical geometry of the EGSnrc simulation. The $898 \mu \mathrm{m}$ ionization chamber differs the most and the $138 \mu \mathrm{m}$ ionization chamber differs the least from the 
simplifying assumptions used for the analytic approximation.

In the left panel of Fig. 7 the histogram for the chamber with the $898 \mu \mathrm{m}$ gap does not overlap the histograms for the chambers with the 138 and $268 \mu \mathrm{m}$ gaps for several reasons, one of which is that some betas, after crossing the gap, multiple scatter through large angles in the copper tube and return to the liquid argon. An example is the simulated track in Fig. 1. The spectrum of energies deposited by those betas which enter the liquid argon is shown in Fig. 8.

\subsection{Drift currents}

A custom simulation [22] of charge production, charge transport, charge loss at the electrodes, and charge loss via recombination (RIR and RDR) was written to compare with our data. We considered not only electrons and positive argon ions but also some other positive charge carrier in addition to argon ions and a small contamination of oxygen molecules which easily attach free electrons to become oxygen molecular ions. The simulation suggests we have little sensitivity to additional positive charge carriers so we do not include this mechanism in the following.

The simulation program assumed the electric field and the drift velocities are purely radial in a cylindrical coordinate system. The gap between the coaxial cylindrical electrodes was divided into cells of uniform steps in radius and the charges in each cell were tracked as a function of time. At the beginning of a simulation run the cells contained only neutral argon and a small contamination of neutral $\mathrm{O}_{2}$. A constant time step was chosen such that the fastest electron drifts less than a quarter of the way across a cell in one time step. In each time step the number of each charge species in a cell changed due to ionization, attachment, recombination, and/or drift (or diffusion) into a neighbor radial cell or, in the case of the radial cell at each end, to an electrode. Then the electric field in each cell was calculated via Gauss' Law with the constraint that the line integral of the electric field equaled the potential across the gap.

The magnitude of the ionization rate-density $D_{i}$ in each radial and axial cell at $\mathcal{E}_{\mathrm{T}}=2200 \mathrm{~V} / \mathrm{mm}$ and ignoring RDR effects was derived from the rate- 
density of the EGSnrc energy deposit in that region divided by $W\left(\mathcal{E}_{\mathrm{T}}\right)=23.6$ $\mathrm{eV}$ per ionization. We use $D_{i}=8.703(8.281,6.467) \times 10^{10}$ ionizations $/ \mathrm{mm}^{3} / \mathrm{s}$ averaged across the gap for the strong source and $D_{i}=3.937(3.810,3.015) \times 10^{9}$ ionizations $/ \mathrm{mm}^{3} / \mathrm{s}$ for the weak source. This can be compared with $D_{i}=$ $6.3 \times 10^{10}$ ionizations $/ \mathrm{mm}^{3} / \mathrm{s}$ at $\eta=4.7$ in the ATLAS liquid argon forward calorimeter at a depth near EM shower maximum at the design center-of-mass energy of $14 \mathrm{TeV}$ and luminosity of $10^{34} \mathrm{~cm}^{-3} \mathrm{~s}^{-1}$ and at $\mathcal{E}_{\mathrm{T}}=2200 \mathrm{~V} / \mathrm{mm}$.

Many of the mechanisms in this simulation are poorly known or not known at all. We employed reasonable descriptions and explored a range of parameters in our attempt to find a set which produced results in agreement with our data.

Of the poorly known parameters, the one of most importance in this study was the positive argon ion mobility. We presumed this single parameter describes the argon ion velocity at all electric fields. This mobility is the critical parameter which determines the threshold ionization rate above which the chamber passes into the space-charge limited regime. Some [23, 24] have suggested that an argon ion may combine with one or more neutral argon atoms to form a singly-charged argon molecular ion. And the possibility of positive charge transport in solid argon [25] suggests that hole transport (charge exchange) may play a role in liquid argon as well. Others [26] suggest that impurity atoms or molecules with an ionization potential lower than argon can capture the charge from the drifting argon ions and the resulting positive ions have a lower mobility than the argon ions. This mechanism suggests that the space-charge threshold will depend on the impurities. So there could be multiple positive charge carriers each with a different mobility. We don't pursue this possibility in this report.

For the free electron drift velocity in liquid argon as a function of electric field we used a parameterization similar to that in reference [27]

$$
v_{e}(\mathcal{E})= \begin{cases}A\left(\mathcal{E} / \mathcal{E}_{a}\right)^{a} & \text { for } 0<\mathcal{E}<\mathcal{E}_{a} \\ B\left(\mathcal{E} / \mathcal{E}_{b}\right)^{b} & \text { for } \mathcal{E}_{a}<\mathcal{E}<\mathcal{E}_{b} \\ C\left(\mathcal{E} / \mathcal{E}_{b}\right)^{c} & \text { for } \mathcal{E}>\mathcal{E}_{b}\end{cases}
$$


where $v_{e}(\mathcal{E})$ is in units of $\mathrm{mm} / \mathrm{s}$ and the electric field $\mathcal{E}$ is in units of $\mathrm{V} / \mathrm{mm}$. The parameters are $\mathcal{E}_{a}=20 \mathrm{~V} / \mathrm{mm}, \mathcal{E}_{b}=2000 \mathrm{~V} / \mathrm{mm}, A=\mu_{e} \mathcal{E}_{a}, B=v_{m} /\left(\mathcal{E}_{0} / \mathcal{E}_{b}\right)^{b}$, $a=1, b=\ln \left(v_{m} / A\right) / \ln \left(\mathcal{E}_{0} / \mathcal{E}_{a}\right), c=0.1, \mu_{e}=5 \times 10^{4} \mathrm{~mm}^{2} / \mathrm{Vs}, v_{m}=4.2 \times 10^{6}$ $\mathrm{mm} / \mathrm{s}$, and $\mathcal{E}_{0}=1000 \mathrm{~V} / \mathrm{mm}$.

Superimposed on the gap current data in Fig. 5 is a parameterization of the shape of the HV Plateau Curve whose free parameters were adjusted to give a good fit in the region where RDR can be neglected. Several phenomenological models were available [28-30]. We used the Birks' Law [31] inspired parameterization of equation 11 where the first term is meant to approximate the ionization from minimum-ionizing betas and the next two terms are meant to approximate successively more densely ionizing betas and delta rays.

$$
P(\mathcal{E})=\frac{\alpha}{1+\mathcal{E}_{1} / \mathcal{E}}+\frac{(1-\alpha) \beta}{1+\mathcal{E}_{2} / \mathcal{E}}+\frac{(1-\alpha)(1-\beta)}{1+\mathcal{E}_{3} / \mathcal{E}}
$$

where $\mathcal{E}$ is the electric field, and $\mathcal{E}_{1}, \mathcal{E}_{2}$, and $\mathcal{E}_{3}$ are characteristic electric fields. In this paper we used $\alpha=0.22, \beta=0.80, \mathcal{E}_{1}=1.5 \mathrm{~V} / \mathrm{mm}, \mathcal{E}_{2}=35 \mathrm{~V} / \mathrm{mm}$, and $\mathcal{E}_{3}=1500 \mathrm{~V} / \mathrm{mm}$. The shape of this parameterization is similar to that used in ref. [18] and [32] at intermediate and high electric fields.

Bulk recombination was parameterized as

$$
D_{r} \equiv-\frac{d n_{+}}{d t} \equiv-\frac{d n_{-}}{d t}=R_{r} n_{+} n_{-}
$$

where $D_{r}$ is the rate of bulk recombination per unit volume, $R_{r}$ is the rate constant, and $n_{+}$and $n_{-}$are the electron and positive argon ion number densities which depend on the rate of high energy particles. The value of $R_{r}$ depends on electric field $\mathcal{E}$. The measurements of the bulk recombination rate constant in reference [33] covered electric fields up to $80 \mathrm{~V} / \mathrm{mm}$. We could not find reasonable agreement between the simulation and our data with such high values. So we used $R_{r} \approx 0.005 \mathrm{~mm}^{3} / \mathrm{s}$ for $\mathcal{E}<200 \mathrm{~V} / \mathrm{mm}$ and $R_{r} \approx 0.001 \mathrm{~mm}^{3} / \mathrm{s}$ for $\mathcal{E}>200 \mathrm{~V} / \mathrm{mm}$.

The attachment rate of electrons to oxygen molecules is a bulk effect similar to the bulk recombination rate of electrons to positive argon ions in equation 12 
and so was parameterized with an electric field dependent rate constant which has been well measured [34-37].

On the other hand the mobility of oxygen molecular ions in liquid argon is very uncertain, particularly near a pressure of 1.0 bar where we operate $[24,38]$. And the rate constant for oxygen molecular ion recombination with positive argon ions has not been measured. We use $\mu_{X}=0.078 \mathrm{~mm}^{2} /$ Vs. And we use $k_{X}=e\left(\mu_{+}+\mu_{X}\right) / \epsilon=1.89 \times 10^{-6} \mathrm{~mm}^{3} / \mathrm{s}$ as suggested by R.Holroyd [39].

While our custom simulation reported the full charge and current configurations within the gap, the only observable was the external current. Within the simulation the current was calculated three different ways. We recorded the current at the boundary of the first cell next to the anode and the current at the boundary of the last cell next to the cathode. We also calculated the net production of charges within the chamber after the recombination effects. Because the simulation relaxed to a steady-state all three current measurements should be the same and Maxwell's displacement current within the chamber gaps was zero. These three currents agreed to more than three significant digits.

We ran several checks of our custom simulation. For example, with the HV plateau parameterization set to unity above $\mathcal{E}=0.1 \mathrm{~V} / \mathrm{mm}$, with uniform ionization, with the bulk recombination rate set to the low value of $3 \times 10^{-4}$ $\mathrm{mm}^{3} / \mathrm{s}$, and with no oxygen contamination the simulation returned currents in good agreement with the analytic approximation. The ratio of currents from the simulation still agreed well with the analytic approximation in equations 5 , 6 , and 8 when using the realistic HV plateau curve of equation 11.

The various unknown or poorly known parameters were varied and the simulation currents were then compared with the data in Fig. 5 for the $268 \mu \mathrm{m}$ gap and with the corresponding data for the other two gaps. Ratios of the simulated currents were also compared to the data in Fig. 6. The simulation was also adapted to the HiLum project [40] and the simulation parameters were required to agree with this data as well.

The best value of positive argon ion mobility to one significant digit is $\mu_{+}=$ $0.08 \pm 0.02 \mathrm{~mm}^{2} / \mathrm{Vs}$. The simulation shows rounding near the thresholds in 
rough agreement with the data. The simulation ratio approaches $21.37^{3 / 4}=9.93$ very approximately at low electric fields.

With an oxygen contamination in the simulation consistent with our oxygen analyzer readings the simulated currents showed more degradation than we observe. We obtained simulation results in good agreement with our data when using an oxygen contamination below $0.05 \mathrm{ppm}$.

As the weak source gap crossed into the space-charge regime the simulation was excessively sensitive to many effects. We were unable to find parameters for which the simulation agreed with the data for all three gaps. It is possible that the data itself is also very sensitive to extraneous effects at these low potentials. But our focus is on the behavior at higher potentials where the simulation and the data appear to be dependent only on the processes we have discussed.

\section{Space-Charge Effects}

If there were only RIR effects then the current ratios in Fig. 6 would be constant, independent of potential. The change in this ratio with the gap potential indicates the presence of RDR effects.

The current in each strong source chamber drops with decreasing potential below $V_{c 2}$ relative to its corresponding weak source chamber due to the additional RDR effects. This is seen most clearly in Fig. 6 where the ratio of strong source currents to weak source currents cancels out the RIR effects, leaving only RDR effects as the cause of variations with potential. The potentials over which each gap current drops is sensitive to the positive argon ion mobility. But what we measure directly is the somewhat gradual threshold for entering the space-charge limited regime. The threshold is not as sharp as suggested by the analytic approximation of Section 2. This threshold is softened by the electron-positive argon ion recombination. If the positive ion velocity is not well described by a mobility and/or if there are multiple species of positive charges then our interpretation in terms of a single positive argon ion mobility may be incorrect. In this case we take our value of positive ion mobility as a convenient 
parameter to characterize the space-charge threshold.

At lower potentials each weak source chamber also reaches its threshold (at $V_{c 1}$ ) and enters the space-charge limited regime. At potentials below this threshold the current ratio tends to flatten out again at roughly the predicted value. This confirms our understanding of the space-charge phenomenon we are observing.

\section{Summary}

Our measurement of the stability of the ionization current at the $0.1 \%$ level over a period of more than 10 months in a high radiation field suggests that liquid argon calorimetry is a radiation tolerant technology for long exposures in high rate environments. This exposure corresponds to an integrated luminosity of about $330 \mathrm{fb}^{-1}$ for an ionization chamber at $\eta=4.7$ at a depth in a calorimeter near EM shower max at a distance of about $5 \mathrm{~m}$ from the interaction point.

Of the many measurements of the HV plateau curve (RIR effect) in the literature, the data presented here is of high precision and covers a wide range of potentials. From this data it is evident that our HV currents continue to slowly rise for electric fields above $\mathcal{E}=22 \mathrm{kV} / \mathrm{cm}[20]$ rather than "plateauing" as some suggest. This observation challenges the assumption that the measurement of $W=23.6 \mathrm{eV}$ is at an asymptotic electric field. While our parameterization in equation 11 does approach an asymptotic current, this occurs above our range of electric fields.

It should be noted that HV plateau curves are not universal. That is, these plateau curves are not necessarily the same as those taken with a different beta energy spectrum or with different species of ionizing particles.

The precision of our step currents allows a precise comparison at high potentials where RDR effects are negligible. We see a constant ratio of strong source current to weak source current of $A=21.37$ with good accuracy. At the lowest potentials the ratio of strong source current to weak source current appears consistent with the predicted constant of $A^{3 / 4}=9.94$. 
Between these two extremes the strong source currents deviate from equation 11 at a potential of $27.5(104,1004) \mathrm{V}$ for the $138(268,898) \mu \mathrm{m}$ gap. The weak source step currents deviate from equation 11 around $5.3(21,206) \mathrm{V}$. The current ratios, compared to our analytic approximation or to our simulation, allow us to extract the positive argon ion mobility of $\mu_{+}=0.08 \pm 0.02 \mathrm{~mm}^{2} / \mathrm{Vs}$ at a temperature of $88.0 \pm 0.5 \mathrm{~K}$ and pressure of $1.02 \pm 0.02$ bar. We note that this parameter serves to indicate the threshold for the on-set of space-charge (RDR) effects even if the mechanism of positive charge transport may be more complicated than our simple assumption.

The threshold for entering the space-charge limited regime is not as sharp as suggested by the analytic approximations. The bulk recombination rate constant rounds these sharp thresholds so that deviations from rate independence begin to appear below the threshold, i.e. above the critical potential. We have found that our data are not consistent with the large bulk recombination rate constants reported in reference [33].

Operating a liquid argon calorimeter at ionization rates above the critical value (equation 1 ) will lead to space-charge effects which will degrade the performance. The practical method to forestall the on-set of space charge effects, that is, to raise the threshold ionization rate, is to build a liquid argon calorimeter with smaller gaps.

By measuring the d.c. currents we have determined the behavior of liquid argon ionization chambers in calorimeters faced with the approximately constant bombardment of particles from the most common hadron collisions. When occasionally there is a collision of interest the ionization chamber then produces a transient pulse. The behavior of such pulses on top of this d.c. background is a different story not addressed in this report.

\section{Acknowledgments}

We thank M.Shupe, L.Shaver, E.Kelly, B.Toggerson, C.Reynolds, M.Maher, J.Ronan, and A.Al-Dabbagh for help with various phases of this experiment and 
its analysis. We also thank Sven Menke, Veljko Radeka, and Richard Holroyd for insightful questions and pointers to related work.

This work was supported by the Office of High Energy Physics of the U.S. Department of Energy as part of the U.S. ATLAS Research Program and by Grant no. DE-FG02-04ER41298 from the U.S. Department of Energy.

[1] Lyndon Evans and Philip Bryant (editors), "LHC Machine", 2008 JINST $3 \mathrm{~S} 08001$.

[2] ATLAS Collaboration, "The ATLAS Experiment at the CERN Large Hadron Collider", 2008 JINST 3 S08003.

[3] CMS Collaboration, "The CMS Experiment at the CERN LHC", 2008 JINST 3 S08004.

[4] ATLAS Collaboration, et al, EPJC 70 (2010) 755.

[5] Bruno Rossi, "High-Energy Particles", Prentice-Hall, Inc. Englewood Cliffs, N.J. 1965.

[6] By "asymptotic potential" we mean the potential at which the HV Plateau Curve becomes independent of electric field. It is not clear that there is such a potential. If there is it must be low enough that the argon does not break down or that the cathode does not experience field emission.

[7] We are neglecting "columnar recombination". In the liquid argon calorimeters in ATLAS none of the incident particles are parallel to the drift field. Perhaps more important, most of the ionization comes from shower particles several generations removed from the incident particle. These particles are spread over a wide range of angles so that a negligible number are parallel to the drift field.

[8] J. Rutherfoord, Nucl. Instr. and Meth. A 482 (2002) 156 and references therein. 
[9] E. Fermi, Zeitschrift für Physik 88 (1934) 161; Jack P. Davidson, Jr., Phys. Rev. 82 (1951) 48.

[10] MonoTorr Phase 1 3000, SAES Pure Gas, Inc., San Luis Obispo, California, USA.

[11] Trace Oxygen Analyzer Model FA30111A, Delta F Corporation, 4 Constitution Way, Woburn, MA 01801-1084. Illinois Instruments Model 912, 2401 Hiller Ridge Road, Johnsburg, IL 60050, USA.

[12] CY7-SD7 Temperature Sensor, OMEGA Engineering, INC., One Omega Drive, Stamford, CT 06907, USA.

[13] PX176-025A5V Pressure Transducer, OMEGA Engineering, INC., One Omega Drive, Stamford, CT 06907, USA.

[14] Ortec 710 Quad Bias Supply, Oak Ridge, Tennessee, USA.

[15] HV Power Supply Model 375P and Model 380X, Bertan Associates, Inc. 121 New South Rd., Hicksville, NY 11803 USA.

[16] Keithley 485 Autoranging picoAmmeter, Cleveland, Ohio, USA.

[17] LabVIEW is a product of National Instruments, Austin, Texas, USA.

[18] B. Toggerson, A. Newcomer, J. Rutherfoord, and R.B. Walker, Nucl. Instr. and Meth. A 608 (2009) 238; doi:10.1016/j.nima. 2009.07.013.

[19] http://www.nrc-cnrc.gc.ca/eng/solutions/advisory/egsnrcindex. html This is an extension, primarily to lower energies, of the SLAC software simulation package EGS4.

[20] M. Miyajima, T. Takahashi, S. Konno, T. Hamada, S. Kubota, H. Shibamura, and T. Doke, Phys. Rev. A 9 (1974) 1438 and Erratum in Phys. Rev. A 10 (1974) 1452.

[21] http://physics.nist.gov/PhysRefData/Star/Text/ESTAR.html. Select "unique material" and specify the parameters for liquid argon. 
[22] See section 10 of reference [8] for an earlier description of the computer code used to simulate charge creation, transport, and loss in liquid argon.

[23] Bob L. Henson, Phys. Rev. 135 (1964) A1002.

[24] T.H. Dey and T.J. Lewis, Brit.J.Appl. Phys. (J.Phys. D) 1 (1968) 1019.

[25] W. Spear, P. Le Comber, in: M.L. Klein and J.A. Venables (ed), "Rare Gas Solids, Vol. II" (1976), p. 1120.

[26] M.A. Kirsanov and I.M. Obodovskiy, Probory i Tekhnika Eksperimenta, 2010, No. 2, 31 (in Russian); [Engl. Transl. in Instruments and Experimental Techniques 53 (2010) 185.]

[27] E. Aprile, K.L. Giboni, and C. Rubbia, Nucl. Instr. and Meth. A 241 (1985) 62.

[28] J. Thomas and D.A. Imel, Phy. Rev. A 36 (1987) 614.

[29] J. Thomas, D.A. Imel, and S. Biller, Phys. Rev. A 38 (1988) 5793.

[30] M.L. Andrieux, J. Collot, P.de Saintignon, A. Ferrari, J.Y. Hostachy, and Ph. Martin, Nucl. Instr. and Meth. A 427 (1999) 568.

[31] J. Birks, "Theory and Practice of Scintillation Counting", Pergamon Press, 1964.

[32] Elena Aprile et al., IEEE Trans. Nucl. Sci. 35 (1988) 37.

[33] K. Shinsaka and Y. Hatano, Nucl. Instr. and Meth. A 327 (1993) 7; K. Shinsaka, M. Codama, T. Srithanratana, M. Yamamoto, and Y. Hatano, J. Chem. Phys. 88 (1988) 7529.

[34] M. Adams, P. Buchholz, A. Dahlhoff, D. Freiden, V. Gaertner, M. Grams, M. Haarmann, A. Hergesell, F. Hoelldorfer, G. Ielapi, K. Jakobs, T. Jung, L. Koepke, E. Kuhnert, D. Meder, U. Schaefer, A. Schmidt, Y. Schue, C. Schwarz, W. Walkowiak, D. Weber, D. Wilke, C. Zeitnitz, Nucl. Instr. and Meth. A 545 (2005) 613. 
807

808

809

810

[35] G. Bakele, U. Sowada, and W. Schmidt, J. Phys. Chem. 80 (1976) 2556.

[36] S. Biller et al., Nucl. Instr. and Meth. A 276 (1989) 144.

[37] W. Hofmann, U. Klein, M. Schulz, J. Spengler, and D. Wedener, Nucl. Instr. and Meth. A 135 (1976) 151.

[38] H.T. Davis, Stuart A. Rice, and Lothar Meyer, J. Chem. Phys. 37 (1962) 2470 .

[39] R. Holroyd, Brookhaven National Accelerator Laboratory Chemistry Department, emeritus. Private communication.

[40] A. Glatte et al., Nucl. Instr. and Meth. A 669 (2012) 47. 


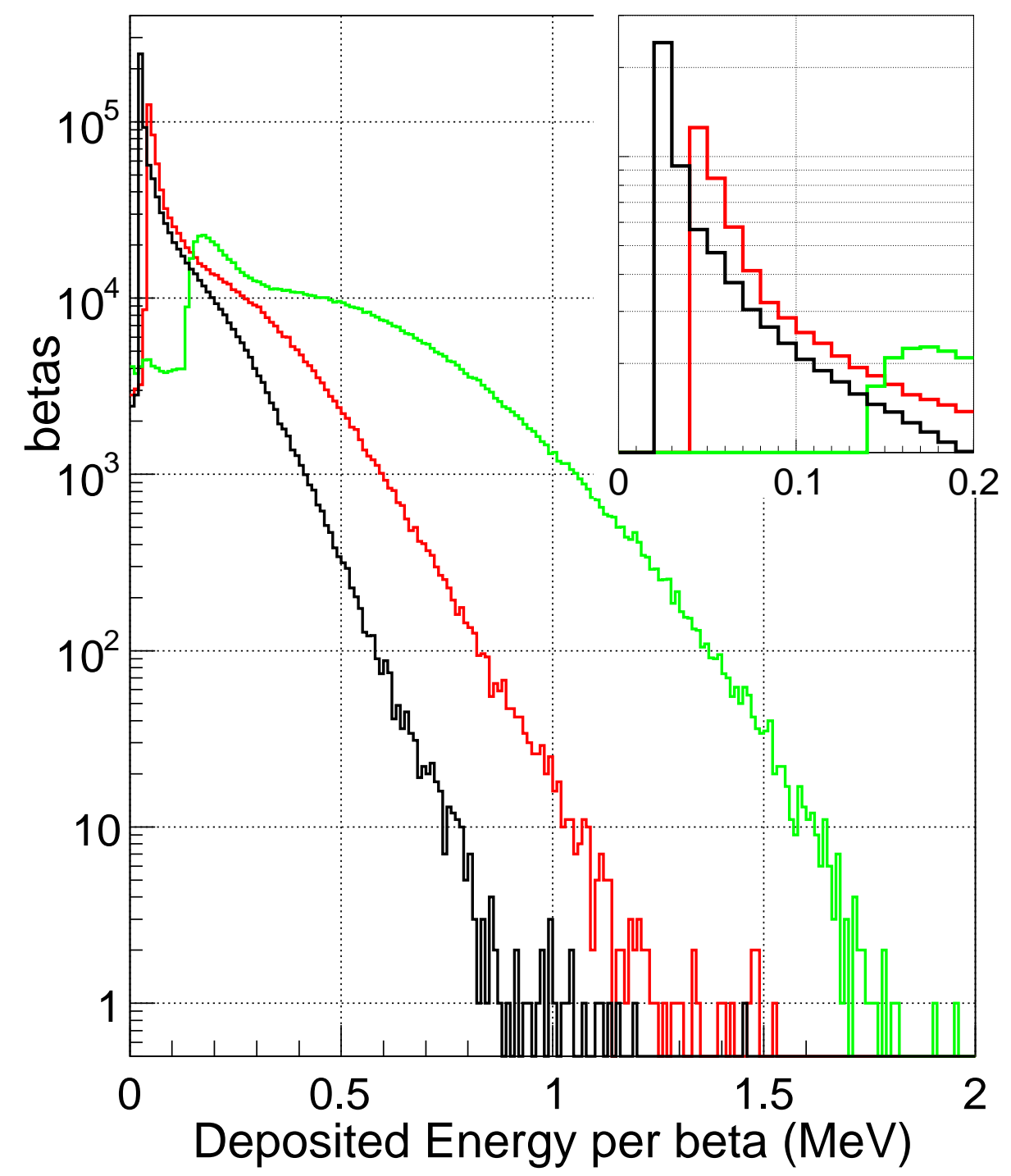

Figure 8: Histogram of the energy deposited in the liquid argon by each simulated beta. The average energy deposit per beta is about $90(162,411) \mathrm{keV}$. For comparison, a beta with kinetic energy of $1.0 \mathrm{MeV}$ travelling in a straight line across the gap (normal to the electrode surfaces) would lose about $27,(52,173) \mathrm{keV}[21]$ in the liquid argon. 